\title{
RDUS
}

Revue de DROIT

UNIVERSITÉ DE SHERBROOKE

Titre : $\quad$ LE RÔLE DU DROIT CIVIL QUÉBÉCOIS ET L'UTILISATION DU DROIT COMPARÉ EN DROIT MARITIME CANADIEN

Auteur(s): $\quad$ André BRAËN

Revue : $\quad$ RDUS, 2013, volume 43, numéro 1-2

Pages: $\quad 561-584$

ISSN : $\quad 0317-9656$

Éditeur : $\quad$ Université de Sherbrooke. Faculté de droit.

URI : $\quad$ http://hdl.handle.net/11143/10222

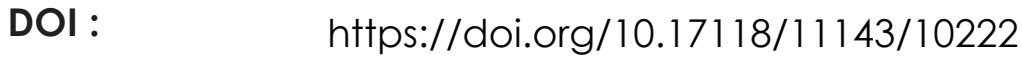


Page vide laissée intentionnellement. 


\title{
ARTICLE
}

\section{LE RÔLE DU DROIT CIVIL QUÉBÉCOIS ET L'UTILISATION DU DROIT COMPARÉ EN DROIT MARITIME CANADIEN}

\author{
par André BRAËN*
}

\begin{abstract}
Compte tenu du caractère international que revêt bien souvent un transport maritime, le droit comparé joue un rôle utile en droit maritime canadien. Par ailleurs, ce dernier exclut l'application par un tribunal, dans un litige où une question maritime est soulevée, de règles de droit provincial. Curieusement et même lorsque ce litige est localisé dans la province de Québec, c'est au moyen d'une approche comparative que le droit civil québécois est appelé à jouer un certain rôle.
\end{abstract}

Often, maritime shipping is international in character and therefore, comparative law plays a useful role in Canadian maritime law. In a maritime dispute before the courts, Canadian maritime law must be applied which also means the exclusion of any provincial rules for deciding the case. Curiously, it is only by utilizing the comparative method that the civil law is called to play a certain role in admiralty matters even when the case arises in Quebec.

Professeur titulaire à l’Université d'Ottawa et avocat. 


\section{SOMMAIRE}

Remarques préliminaires 563

I- Le droit maritime canadien 567

II- L'application du droit provincial et le recours au droit comparé ............................................ 572

III- Le rôle du droit civil......................................... 580

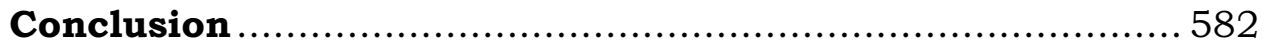


Le rôle du droit civil québécois

(2013) 43 R.D.U.S. et l'utilisation du droit comparé

\section{Remarques préliminaires}

S’interroger sur l'utilisation du droit comparé en droit maritime canadien et sur le rôle qu'y joue le droit civil québécois appelle quelques remarques préliminaires. D'abord et au plan des définitions, le droit maritime s'entend traditionnellement du droit appliqué par les juridictions spécialisées que sont les tribunaux

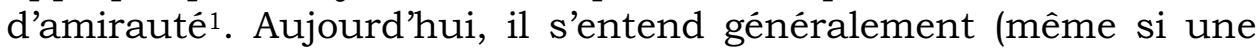
définition plus englobante peut lui être accolée ${ }^{2}$ ) du droit applicable au transport et à la navigation maritimes. Quant à l'expression "droit maritime canadien ", elle est d'origine législative $^{3}$ et elle renvoie à un contenu dont la croissance illimitée semble être la norme. Nous y reviendrons.

Puis, les transports maritimes sont très fréquemment des transports internationaux et les périls de mer, quant à eux, ignorent la géographie politique de sorte que plusieurs lois pourraient prétendre être appliquées à une même expédition, créant en cela une opposition très vive entre le caractère national de la législation maritime adoptée par un État et la réalité du transport maritime. Aussi, il n'est pas surprenant de constater que ce domaine du droit a fait, très tôt, l'objet de plusieurs tentatives d'uniformisation et d'unification, ou en tous les cas, d'harmonisation donnant ainsi

1. On parle alors du droit de l'amirauté pour mieux l'opposer au droit maritime. Mais aujourd'hui la distinction n'est plus qu'une question de sémantique.

2. Ultimement, le droit maritime engloberait les règles juridiques applicables à toutes les activités se déroulant dans un milieu maritime. Voir André BRAËN, Le droit maritime au Québec, Montréal, Wilson \& Lafleur, 1992, pp. 1-4. Voir aussi : Michel MoRIN, "Le droit maritime : diversité ou fragmentation ", (1997) XV Annuaire de droit maritime et océanique, 281; René RodiĖre et Emmanuel du Pontavice, Droit maritime, 10e éd., Paris, Dalloz, 1986, p. 1; William TETLEY, International Maritime and Admiralty Law, Cowansville, Éditions Yvon Blais, 2002, pp. xiii-xiv; Thomas J. Schoenbaum et A.N. Yiannopoulos, Admiralty and Maritime Law, Charlottesville, The Michie Company, 1984, pp. 1-2. Pour une illustration de cette définition englobante, voir : Jean-Pierre BEURIER (dir.), Droits maritimes, 2e éd., Paris, Dalloz, 2008.

3. Loi sur les Cours fédérales, L.R.C. 1985, c. F-7, art. 2. 
suite à une volonté de rechercher une solution commune ${ }^{4}$. Plusieurs organisations internationales, gouvernementales comme la CNUCED $^{5}$ et non gouvernementales comme le $\mathrm{CMI}^{6}$, pour ne nommer que celles-là ${ }^{7}$, s'y consacrent.

Si la règle de droit maritime peut donc aussi se développer au plan international, plusieurs écueils freinent toutefois ce mouvement $^{8}$. Ainsi, s'il est vrai que de nombreuses conventions multilatérales existent dans le domaine maritime, quelquefois traitant du même sujet et se dédoublant, certaines ont fait l'objet de ratification de la part d'un nombre limité d'États; d'autres ont subi des modifications importantes, lesquelles n'ont pas toutes été adoptées par les États... de sorte qu'une certaine confusion s'est installée9. Par ailleurs, il n'existe pas de mécanisme international d'unification de l'interprétation des textes maritimes. Enfin, il faut mentionner l'inflation dans la production des normes juridiques, surtout dans le domaine de la sécurité maritime, énoncées dans d'innombrables textes que les organisations spécialisées, en parti-

4. L'uniformisation du droit maritime au plan international s'entend d'un processus d'harmonisation des règles compte tenu de la diversité des traditions juridiques. C'est le résultat qui compte. Voir Peter GRIGGS, "Uniformity of Maritime Law - an International Perspective ", (1993) 73 Tul.L.R. 1551. Voir aussi pour une analyse plus détaillée Nathalie VÉzINA (dir.), Le droit uniforme : limites et possibilités, Cowansville, Éditions Yvon Blais, 2009.

5. Conférence des Nations-Unies sur le commerce et le développement (www.unctad.org).

6. Comité maritime international (www.comitemaritime.org).

7. Pour un aperçu de toutes ces organisations, voir Edgar GoLD, Aldo CHIRCOP et Hugh KInDRED, Maritime Law, Toronto, Irwin Law, 2003, pp. 7598.

8. Pierre Bonassies et Christian ScApel, Droit maritime, Paris, L.G.D.J., 2006, p. 16. Voir aussi P. GRIGGS, préc., note 4.

9. L'exemple le plus patent est celui du régime juridique applicable à la responsabilité du transporteur maritime de marchandises. C'est là un domaine où cohabitent les Règles de La Haye, les Règles de La HayeVisby, la Convention de Hambourg et la toute récente, celle de Rotterdam. Toutes ont la prétention d'unifier les règles dans ce domaine. Voir Marel KATSIVELA, "Overview of Ocean Carrier Liability Exceptions Under the Rotterdam Rules and the Hague-Hague/Visby Rules ", (2010) 40 R.G.D.413. 
Le rôle du droit civil québécois
(2013) 43 R.D.U.S. l'utilisation du droit comparé
en droit maritime canadien

culier 1'OMI10, proposent aux États et laquelle ajoute à cette confusion et constitue de façon paradoxale un frein à l'uniformité11. Une exception notable à cet éparpillement existe et vient de la volonté des acteurs privés de l'industrie maritime d'appliquer systématiquement dans leurs relations contractuelles les Règles d'York et d'Anvers au règlement des avaries communes plutôt que les législations nationales ${ }^{12}$.

Selon Patrick Glenn, le raisonnement juridique est un raisonnement essentiellement comparatif 13 . Le droit comparé pour sa part permet un jugement de valeur entre les règles de diverses juridictions ou entre diverses périodes de temps à l'intérieur de la même juridiction. Il offre donc au juriste un outil fort utile pour moderniser le droit et l'harmoniser à l'environnement qui est le sien. Au plan interne, il arrive donc que le tribunal dans son travail d'interprétation fasse expressément référence au droit étranger. Un cas connu est celui touchant l'interprétation des droits et libertés fondamentaux ${ }^{14}$. Il n'est pas rare également qu'un regard comparatiste s'attarde, que ce soit en droit civil ou en common law, au régime du voisin participant à la même tradition juridique $^{15}$. Ce regard comparatiste devrait être particulièrement de mise lorsqu'il s'agit d'interpréter des textes émanant de conventions internationales qui ont été incorporées en droit interne. Il

10. Organisation maritime internationale (www.imo.org).

11. Voir Philippe BoIsson, "La problématique des normes ", (1998) XVI Annuaire de droit maritime et océanique, 175.

12. Voir Julian COOKE et Richard CORNAH (éd.), Lowndes \& Rudolph: The Law of General Average and The York-Antwerp Rules, 13e éd., Londres, Sweet and Maxwell, 2008. Quant aux textes législatifs traitant de l'avarie commune, on pourra consulter la Loi sur l'assurance maritime, L.C. 1993, c. 22, art. 65, 72, 77 et 79 et même les art. 2599-2603 du Code civil du Québec.

13. Patrick GLEnN, "Le droit comparé et la Cour suprême du Canada " dans Ernest CAPARRos (dir.), Mélanges Louis-Philippe Pigeon, Montréal, Wilson \& Lafleur, 1989, pp. 197-200.

14. Par exemple voir R. c. Big M Drug Mart Ltd., [1985] 1 R.C.S. 295.

15. Voir Louis LEBEL, "Les cultures de la Cour suprême du Canada: vers l'émergence d'une culture dialogique? " dans Convergence, concurrence et harmonisation des systèmes juridiques. Les journées Maximilien Caron 2008, Montréal, Éditions Thémis, 2009, p. 3. 
faut alors assurer une certaine uniformité à l'interprétation de ces textes. Une interprétation trop locale ou régionaliste viendrait mettre en péril l'unité recherchée ${ }^{16}$. Mais cette préoccupation peut ne pas être présente chez un tribunal qui alors n'hésitera pas à faire prévaloir sa propre interprétation. À cet égard, il faut saluer l'existence au plan doctrinal d'ouvrages de droit maritime dont la méthode comparative constitue la caractéristique fondamentale, en particulier ceux du professeur William Tetley ${ }^{17}$.

Compte tenu de ce qui précède, on imagine facilement le rôle utile que peut jouer en droit maritime canadien la méthode comparative. Curieusement et pour les raisons que nous allons expliquer, l'utilisation du droit civil québécois en droit maritime canadien s'inscrit à l'intérieur de cette approche comparatiste que le tribunal reste toujours libre d'adopter. En effet, le droit civil québécois ne peut prétendre être une source de droit substantif, ni même de droit supplétif pour solutionner un litige maritime et s’il est utilisé, ce ne sera qu’à titre de support comparatif. Et en-

16. Voir par exemple les propos de Lord McMillan au sujet de l'interprétation qu'il convient de donner à la Convention internationale relative à l'unification de certaines règles en matière de Connaissement (Règles de La Haye 1924), 25 août 1924, (1937) 120 S.D.N. 125 dans Stag Line Ltd c. Foscolo, Mango, (1932) A.C. 328, p. 350 et aussi ceux du j. Harrington dans Société Telus Communications c. Peracomo Inc., 2011 C.F. 494, par. 62. Dans ce dernier cas, il s'agissait d'interpréter certaines dispositions de la Convention de 1976 sur la limitation de la responsabilité en matière de créances maritimes, telle qu'intégrée dans la Loi sur la responsabilité en matière de créances maritimes, L.C. 2001, c. 6, art. 24 et annexe 1. Cette décision a été récemment entérinée par la Cour d'appel fédérale qui a rappelé que l'interprétation de conventions internationales exige que l'on se rapporte non pas à des précédents nationaux mais plutôt à des principes généraux largement acceptés : voir 2012 CAF 199, par. 53 (j. Gauthier et j. Trudel).

17. L'analyse du professeur Tetley s'élabore en tenant compte du droit anglais, du droit français, du droit canadien (y inclus le droit civil québécois) et du droit américain. Voir: William TetLey, Maritime Liens and Claims, 2e éd., Cowansville, Éditions Yvon Blais, 1998; International Maritime and Admiralty Law, Cowansville, Éditions Yvon Blais, 2002; International Conflict of Laws, Cowansville, Éditions Yvon Blais, 1994; Marine Cargo Claims, 4e éd. (2 vol.), Cowansville, Éditions Yvon Blais, 2008. 
Le rôle du droit civil québécois

(2013) 43 R.D.U.S. et l'utilisation du droit comparé

en droit maritime canadien

core là, on pourra toujours se demander quelle est son utilité réelle sur ce plan.

\section{I- Le droit maritime canadien}

Lorsqu'un litige devant un tribunal canadien soulève une question maritime ou d'amirauté, c'est nécessairement du droit maritime canadien que doit provenir la solution ${ }^{18}$. Le critère permettant d'établir si une question examinée relève du droit maritime canadien requiert que cette question soit entièrement liée aux affaires maritimes au point de constituer légitimement du droit maritime canadien 19 . Les termes "amirauté " et "maritime " doivent être interprétés libéralement et dans le contexte moderne du commerce et des expéditions par eau ${ }^{20}$.

La Cour fédérale du Canada est le tribunal d'amirauté du Canada chargé d'appliquer de façon concurrente avec les tribunaux des provinces le droit maritime canadien ${ }^{21}$. L'expression "droit maritime canadien " a fait l'objet d'une définition plutôt laborieuse par le Parlement canadien. Elle renvoie aux règles dont l'application relevait de la Cour de l'Échiquier du Canada, en sa qualité de juridiction de l'Amirauté " ou qui en aurait relevé si ce tribunal avait eu, en cette qualité, compétence illimitée en matière maritime et d'amirauté ${ }^{22}$. Cette référence législative à une compétence illimitée s'explique pour des raisons historiques. En bref, qu'il suffise de dire qu'en Angleterre, les règles du droit maritime

18. C'est là l'approche mise laborieusement de l'avant par la Cour suprême du Canada. Voir ITO International Terminal Operators Ltd. c. Miida Electronics Inc., [1986] 1 R.C.S. 752; Q.N.S. Paper Co. c. Chartwell Shipping Ltd., [1989] 2 R.C.S. 683; Whitbread c. Walley, [1990] 3 R.C.S. 1273; Monk c. Island Fertilizers Ltd., [1991] 1 R.C.S. 779, Bow Valley Husky (Bermuda) Ltd. c. Saint John Shipbuilding Ltd., [1997] 3 R.C.S. 1210 et Succession Ordon c. Grail [1998] 3 R.C.S. 437. Pour une analyse détaillée de ce raisonnement voir : André BRAËN, préc., note 2, pp. 86-165.

19. ITO International Terminal Operators Ltd. c. Miida Electronics Ltd., préc., note 18, pp. 774-776 (j. McIntyre).

20. Monk c. Island Fertilizers Ltd., préc., note 18, p. 800 (j. Iacobucci).

21. Loi sur les Cours fédérales, préc., note 3, art. 22(1).

22. Id., art. 2 . 
furent développées tant par la cour de l'Amirauté que par les tribunaux de common law, ces derniers contestant la compétence qui avait été attribuée à l'Amiral dès le 14e siècle. C'est la fusion de ces tribunaux ordonnée par le Parlement britannique en $1873^{23}$ ainsi que l'attribution d'une compétence en amirauté au tribunal de droit commun qui mettront fin à ce conflit et aujourd'hui, le droit maritime anglais s'entend simplement des règles et principes de common law appliqués aux affaires maritimes ${ }^{24}$.

Au Canada, l'attribution d'une compétence en amirauté d'abord à la Cour de l'Échiquier se fit au moyen d'un renvoi au droit appliqué originalement par l'Amirauté britannique ${ }^{25}$, donc à une juridiction contestée et à un droit aux pourtours incertains ${ }^{26}$. C'est ce qui explique le renvoi, en ce qui concerne son successeur la Cour fédérale, à une compétence que le législateur prétend "illimitée "27. Selon la Cour suprême du Canada et en termes de contenu, le droit maritime canadien ainsi défini s'entend de (1) la législation fédérale adoptée en matière de navigation et de marine marchande, (2) des règles et principes émanant de la compétence exercée par la Cour d'Amirauté anglaise en 1934 (l'année de la dernière adoption par voie législative au Canada du droit anglais en matière d'amirauté28) et (3) des règles et principes que les tribunaux de common law anglais appliquent aux litiges maritimes tels

23. Supreme Court of Judicature Act, (1873) 36 \& 37 Vict., c. 66 (R-U).

24. The Tojo Maru, (1972) A.C. 242, p. 291 (C. L.). Voir aussi André BRAËN, "Le droit maritime au Québec à l'aube de la Codification ", (1985) 16 R.G.D. 429.

25. Cette compétence était fondée sur le Colonial Courts of Admiralty Act, (1890) 53-54 Vict., c. 27 (Imp.). Voir la Loi sur l'Amirauté, (1891) 54-55 Vict., c. 29 qui désigne la Cour de l'Échiquier du Canada comme Cour coloniale d'amirauté ayant et exerçant toute la juridiction, les pouvoirs et l'autorité conférés par la législation impériale. En 1934, le Parlement canadien confirma le rôle de la Cour de l'Échiquier dans sa juridiction d'amirauté et lui attribua encore une fois une compétence déterminée par renvoi à celle exercée par la Haute Cour d'Angleterre. Voir : Loi sur l'Amirauté, S.C. 1934, c. 31, art. 3(1).

26. L.H. LAING, "Historic Origins of Admiralty Jurisdiction in England ", (1946-47) 45 Mich. L.R. 163.

27. Préc., note 22.

28. Préc., note 25. 
que ces derniers sont reçus au Canada29. L'ordre doit être respecté et la solution au litige maritime doit donc être recherchée dans la législation fédérale, ou en l'absence de règles fédérales, dans les principes mis de l'avant par la Cour d'Amirauté anglaise ou à défaut, dans les règles que la common law anglaise applique à ces litiges et telles que ces règles sont reçues dans la jurisprudence canadienne. Ce n'est qu'exceptionnellement, comme on le verra, qu'un plaideur pourra invoquer l'application du droit provincial.

Le droit maritime canadien s'entend d'un corps de règles qui relève exclusivement, selon la Cour suprême du Canada et sans que cette dernière ne l'explique clairement à notre avis, de la compétence législative du Parlement canadien en matière de navigation et de marine marchande ${ }^{30}$. L'approche du tribunal a eu comme conséquence, même si curieusement il affirme que son approche reste limitée par le partage des pouvoirs ${ }^{31}$, de reconnaître au plan constitutionnel et en pratique une compétence législative maritime quasiment illimitée au Parlement canadien. Au départ et s'agissant d'en identifier le contenu, la compétence maritime du Parlement canadien avait fait l'objet d'une interprétation libérale, surtout dans ses aspects techniques; mais elle connaissait aussi des limitations importantes en distinguant, par exemple, entre les entreprises de navigation intraprovinciales (relevant de l'autorité provinciale) et les entreprises extraprovinciales (relevant de l'autorité fédérale) ${ }^{32}$.

29. ITO International Terminal Operators Ltd. c. Miida Electronics Inc., préc., note 18.

30. Loi constitutionnelle de 1867, 30 \& 31 Vict., c. 3 (R-U), art. 91(10). Voir André BRAËN, "La compétence maritime du Parlement canadien et ses conséquences sur l'application du Code civil ", (1986) 31 R. D. McGill, 369.

31. Dans l'arrêt ITO International Terminal Operators Ltd., préc., note 18, la cour précise qu'en déterminant si une affaire donnée soulève une question maritime ou d'amirauté, elle doit éviter d'empiéter sur ce qui constitue de par son caractère véritable une matière relevant essentiellement de la compétence provinciale (j. McIntyre pp. 774-775). Voir aussi la dissidence de la juge L'Heureux-Dubé dans l'arrêt Monk c. Island Fertilizers Ltd., préc., note 18 , pp. 801 et ss.

32. André BRAËN, "De l'effet relatif du contrat maritime ou de la relative uniformité du droit maritime canadien ", (2001) 31 R.G.D. 473, pp. 493-500. 
Aujourd'hui, il suffit d'établir une connexité maritime à une question pour en faire relever le sujet de la compétence législative fédérale. Par exemple, celle-ci a été confirmée par la jurisprudence à l'égard de la responsabilité de l'entrepositaire, de celle du mandataire d'un affréteur, de celle découlant de la navigation de plaisance et de toutes questions afférentes, de l'indemnisation de la perte économique, de la négligence dans le contexte maritime... ${ }^{33}$ La qualification d'une question comme étant maritime ${ }^{34}$ a donc comme conséquence de faire relever le sujet de la question de la compétence législative fédérale. Or, puisque l'industrie maritime constitue un secteur d'activités plus vaste que le simple transport de marchandises par eau, les activités terrestres et les activités proprement maritimes s'interpénétrant et se complétant, ce sont finalement toutes les questions relatives à la responsabilité contractuelle et extracontractuelle de tous les intervenants en matière maritime qui doivent trouver leur solution juridique dans le droit maritime canadien et qui relèvent conséquemment de la compétence législative maritime du Parlement.

Pourtant, on aurait cru que ces questions purement de droit privé touchant la responsabilité contractuelle et délictuelle relèvent de la compétence législative d'une province en matière de propriété et droits civils ${ }^{35}$. C'est au moyen de l'attribution d'une compétence judiciaire, que le Parlement prétend illimitée, que la Cour suprême du Canada en est venue à reconnaître une compétence législative pratiquement illimitée au même Parlement. Or, comment un organe législatif dont la souveraineté est nécessairement limitée dans un cadre fédéral peut-il attribuer une compétence judiciaire illimitée?

Voir la Loi constitutionnelle de 1867, préc., note 30, art. 92(10). Mais voir infra note 54 et la récente décision de la Cour suprême du Canada dans l'arrêt Tessier Ltée c. Québec (CSST), 2012 CSC 23.

33. André BRAËN, préc., note 32, p. 496.

34. Pour un exposé de cette démarche voir André BRAËN, préc., note 32, pp. 486-493.

35. Loi constitutionnelle de 1867, préc., note 30, art. 92(13). 
Nous avons identifié deux exceptions à ce courant jurisprudentiel. D'abord, la responsabilité découlant d'activités nautiques comme le plongeon et la natation a été jugée comme ne comportant pas de connexité maritime et donc, comme relevant de l'autorité législative de la province ${ }^{36}$. Puis le second cas est tiré de l'arrêt Isen c. Simms ${ }^{37}$. Dans cette affaire, une embarcation est sortie de l'eau après une journée de navigation de plaisance sur un lac ontarien. Elle est ensuite placée sur une remorque qui est conduite dans un stationnement situé tout près du lac. Avant de transporter l'embarcation sur la route, le propriétaire du bateau tente d'assujettir le couvercle du moteur au moyen d'un câble élastique. Celui-ci glisse de ses doigts et frappe son ami en le blessant sérieusement à l'œil. L'ami et son épouse intentent une action en dommages-intérêts de deux millions et demi de dollars contre le propriétaire. La responsabilité découlant de la négligence du propriétaire de bateau est-elle une question maritime? Pour la Cour suprême du Canada, l'assujettissement du couvercle du moteur du bateau en vue de son transport sur la route n'a rien à voir avec la navigation de celui-ci sur l'eau et tout à voir avec la préparation du bateau en vue de son transport sur les routes de l'Ontario. Ce n'est donc pas une question maritime régie par le droit maritime canadien et c'est le droit ontarien qui doit trouver application. Il faut noter ici qu'avoir jugé cette affaire comme étant, au contraire, une question maritime, la Loi sur la responsabilité en matière maritime ${ }^{38}$ se serait alors appliquée. Or, celle-ci prévoit dans de tels cas une limitation de responsabilité d'un million de dollars en faveur du propriétaire de l'embarcation ${ }^{39}$. Si l'accident s'était produit alors que la remorque était encore dans

36. Dreifelds c. Burton, Ont. CT. Gen. Div. no 8339/94, le 10 avril 1996 et Ont. C.A. no C2456 \& 24580, le 6 mars 1998. Voir aussi Corcovado Yachts Charters Ltd. c. Foreshore Projects Ltd., C.F., no T-153-98, le 9 février 1998.

37. [2006] 2 R.C.S. 349.

38. L.C. 2001 , c. 6.

39. Id., art. 28. Voir maintenant L.C. 2009, c. 21, art. 3. 
l'eau ou sur la grève, la décision eut-elle été la même? Quelques mètres auraient alors suffi pour changer la nature du litige! 40

\section{II- L'application du droit provincial et le recours au droit comparé}

La Cour fédérale a été établie par le Parlement canadien en vue d'une meilleure application des lois du Canada ${ }^{41}$. Le droit maritime canadien, qu'elle est chargée d'appliquer de façon concurrente avec les tribunaux provinciaux ${ }^{42}$, est un ensemble de règles de droit fédéral, duquel est exclu le droit d'une province. Dans l'arrêt ITO International Terminal Operators ${ }^{43}$, la Cour suprême du Canada a identifié le contenu du droit maritime canadien applicable par la Cour fédérale pour déterminer la responsabilité délictuelle et contractuelle d'un entrepositaire du port de Montréal chez qui des marchandises avaient été volées après leur transport maritime. En l'absence de législation fédérale ou de règles de droit maritime britannique, ce sont les règles et principes que les tribunaux de common law appliquent en Angleterre en semblables matières et tels que ces règles et principes sont reçus dans la jurisprudence canadienne ${ }^{44}$ et non pas le droit civil québécois. L'utilisation du droit anglais en droit maritime canadien ne vise donc pas des fins comparatives, mais sert au contraire simplement à confirmer la solution canadienne ou en cas d'absence de règles de droit canadien (législation fédérale ou jurisprudence) à combler ce vide. La solution appliquée tant par les cours fédérales que des provinces empruntera alors au droit anglais ${ }^{45}$.

40. Peut-on imaginer que la cour écartait au moyen de sa décision une limitation de responsabilité qu'elle jugeait peut-être insuffisante compte tenu du montant réclamé par le plaignant?

41. $\quad$ En accord avec l'art. 101 de la Loi constitutionnelle de 1867.

42. Préc., note 21.

43. Préc., note 18.

44. Id., p. 776.

45. Voir concernant le principe de limitation de la responsabilité de l'armateur Société Telus Communications c. Peracomo Inc., 2011 CF 494; concernant le connaissement Kuehne \& Nagel Ltd. c. Agrimax Ltd., 2010 CF 1303; Alcoa Inc. c. CP Ships (UK) Ltd., 2007 ONCA 686; Shtutman c. Oceane Marine Shipping Inc., 2005 CF 1471; Sea Link Marine Services c. 
Le rôle du droit civil québécois

et l'utilisation du droit comparé

en droit maritime canadien

En effet, le droit maritime canadien constitue pour la Cour suprême un droit uniforme qui s'applique partout au Canada et les tribunaux des provinces, lorsqu'ils sont saisis de litiges maritimes, doivent aussi appliquer ce droit maritime canadien. Selon la Cour suprême du Canada dans l'arrêt Q.N.S. Paper Co. c. Chartwell Shipping Ltd. ${ }^{46}$, c'est dans la common law et non dans le droit civil québécois que doit puiser la Cour supérieure du Québec pour déterminer si le mandataire d'un transporteur maritime qui exerce dans cette province est tenu de divulguer l'identité du mandant afin d'éviter d'engager sa responsabilité personnelle envers les tiers. Pour la cour, l'existence de règles de droit maritime uniformes est nécessaire à cause de la nature même des activités relatives à la navigation et aux expéditions par eau, telles qu'elles

Doman Forest Products Ltd., 2003 FCT 712; Timberwest Forest Limited c. Gearbulk Pool Limited et al., 2003 BCCA 39; concernant le remorquage Regina c. Motor Vessel "Katy L ", Gowland Towing Ltd et al., 2010 BCPC 30; Hamilton Marine \& Engineering Ltd. C. CSL Group Inc., (1995) F.C.J. 739; concernant une charte-partie H. Paulin \& Co. Ltd c. A Plus Freight Forwarder Co. Ltd., 2009 CF 727; Pantainer Ltd. c. 996660 Ontario Ltd., (2000) F.C.J. 334; Rio Tinto Shipping (Asia) Pte Ltd. c. Korea Line Corporation, 2008 CF 1376; Canadian Pacific Forest Products c. Termar Navigation Co., [1998] 2 C.F. 328; Alcan c. Unican, (1996) F.C.J. 843; Champion International c. The Sabina, 2002 FCT 1122; Halla Merchant Mariner c. Portserv, (1997) F.C.J. 219; Melsa International c. Adecon, (1997) F.C.J. 445; concernant la responsabilité du transporteur maritime Canadian Forest Products Ltd. c. B.C. Rail Ltd., 2005 BCCA 369; Riva Stahl Gmbh c. Combined Atlantic Carriers Gmbh (1999) 243 N.R. 183 (CAF); Wirth c. Belcan, (1996) F.C.J. 603; Elders Grains Co. C. Ralph Misener (The), 2003 CF 837; concernant les abordages North King Lodge Limited c. Gowlland Towing Ltd, 2005 BCCA 557; North Ridge Fishing Ltd. c. The Prosperity et al., (2002) B.C.J. 1526; Laichkwiltach Enterprises Ltd. c. F3V Pacific Faith (Ship), 2007 BCSC 1852; Omega Salmon Group Ltd. c. Pubnico Gemini 2007 BCCA 33; R. c. Cloutier, 2007 QCCQ 13533; Nordholm I/S c. Canada (1996) F.C.J. 13; concernant les privilèges et saisies-arrêts Norcan Electrical Systems Inc. c. Feeding Systems A/S et al., 2003 FCT 702; Eleonor Soren Toubro (The) [1996] 3 C.F. 422; Zhoushan Zhongchang Shipping Co. c. Handybulk Shipping Ltd., 2004 CF 1135; Whyte c. Sandpiper VI (The), 2002 FCT 572; Richardson International Ltd. c. Zao RPK "Starodubskoe", 2002 FCT 482 et Pacific Tractor Rentals (V.I.) Otd. c. Palaquin (The), (1996) F.C.J. 836.

46. Préc., note 18. 
sont exercées au Canada ${ }^{47}$. Bon nombre des règles de droit maritime sont le produit de conventions internationales et les droits et obligations juridiques de ceux qui se livrent à la navigation et aux expéditions par eau ne devraient pas changer de façon arbitraire suivant l'endroit où ils se trouvent. Il faut toutefois noter que dans toute cette jurisprudence ${ }^{48}$, la Cour suprême n'analyse d'aucune façon les inconvénients que l'application du droit civil en matière maritime a pu causer dans le passé.

L'inclusion de règles de droit provincial, lesquelles peuvent différer d'une province à l'autre, rendrait donc le droit maritime incertain selon la Cour suprême du Canada. Mais un recours aux lois provinciales reste-t-il quand même possible en droit maritime canadien? Dans l'arrêt Succession Ordon c. Grail ${ }^{49}$, le même tribunal précise la situation exceptionnelle où le droit provincial pourrait s'appliquer à une action fondée sur la négligence en droit maritime canadien. C'est en fonction d'une approche en quatre volets qu'il faut déterminer si la loi provinciale peut ainsi s'appliquer50 :

a) Premièrement, le tribunal doit déterminer si la question soulevée relève de la compétence fédérale exclusive sur la navigation et les expéditions par eau. Compte tenu de l'analyse qui précède, il y a peu de chances qu'une question qualifiée de maritime ne relève pas de la compétence fédérale.

47. Id. Voir aussi National Gypsum Co. c. Northern Sales Ltd., [1964] R.C.S. 144; Intermunicipal Realty and Development Corp. c. Gore Mutual Ins. Co., [1978] 2 C.F. 691 et Associated Metals and Minerals Corp. c. Le navire "Evie W.", [1987] 2 C.F. 710 ainsi que la jurisprudence citée à la note 18. Dans Succession Ordon c. Grail, préc., note 18, la Cour suprême avance que les questions maritimes font souvent intervenir une multiplicité d'autorités législatives. Le souci d'uniformité constitue donc un motif qui explique pourquoi, dans le cadre d'une action pour négligence en matière maritime, on ne saurait permettre l'application des lois provinciales de portée générale (p. 503, j. Iacobucci et j. Major).

48. Id.

49. Préc., note 18.

50. Id., par. 73 et ss. 
b) Dans l'affirmative, le deuxième volet consiste à déterminer si la loi provinciale, dont la partie cherche à se prévaloir, a un pendant en droit maritime canadien. Si une règle analogue existe - ce peut être une disposition législative fédérale, une règle de droit maritime britannique ou encore, une règle appliquée par les tribunaux de common law en matière maritime - c'est celle-ci qui doit s'appliquer.

c) S'il n'existe pas une telle règle, le troisième volet consiste pour le tribunal à déterminer s'il convient de modifier le droit maritime canadien non législatif pour remédier à cette lacune ${ }^{51}$.

d) Finalement et si une réforme du droit par les tribunaux n'est pas justifiée, le tribunal doit déterminer si la disposition législative provinciale est constitutionnellement applicable. Pour la cour, la loi provinciale de portée générale sera inapplicable dans un contexte faisant intervenir les règles relatives à la négligence du droit maritime si son application a pour effet de régir indirectement le droit maritime fédéral en matière de négligence.

L'arrêt Succession Ordon c. Grail rend-il possible l'application éventuelle du droit provincial en matière maritime ou le principe de l'exclusivité des compétences rend-il le droit provincial inapplicable? Dans cet arrêt, les juges Iacobbucci et Major ont tout de même précisé que certaines lois provinciales pourraient s'appliquer en matière maritime, en particulier celles réglant la procédure suivie par les tribunaux ou des lois fiscales ${ }^{52}$. Un auteur avance qu'il existe une "périphérie maritime " de laquelle on

51. En tenant compte des principes de réforme judiciaire élaborés par la Cour suprême pour refléter les changements économiques, moraux et sociaux du Canada et ainsi assurer l'évolution du droit. Voir Watkins c. Olafson, [1989] 2 R.C.S. 750 et R. c. Salituro, [1991] 3 R.C.S. 654.

52. Préc., note 18 , p. 499. À notre avis, puisque les tribunaux provinciaux possèdent une compétence concurrente en droit maritime canadien, il allait de soi que la Cour suprême ne puisse que reconnaître l'application des règles provinciales en matière de procédure judiciaire à moins d'anéantir cette compétence. 
ne pourrait évacuer le droit provincial53. De même, il faut mentionner que la Cour suprême du Canada tend, depuis peu, à appliquer avec plus de retenue la doctrine de l'exclusivité des compétences. Une application trop rigoureuse de cette doctrine serait, selon elle, contraire au principe du fédéralisme souple que promeuvent par ailleurs les doctrines du caractère véritable, du double aspect et de la prépondérance fédérale ${ }^{54}$. À cet égard, des lois provinciales d'application générale pourraient-elles ainsi trouver application dans le domaine maritime ${ }^{55}$ ? La question mérite d'être posée quoique le cas échéant, il ne faudrait pas que de telles lois entravent le contenu essentiel de la compétence maritime fédérale ${ }^{56}$. Bref, le brouillard persiste et le plaideur de naviguer dans des eaux troubles 57 .

Précisons que très récemment, la Cour suprême est revenue à une démarche plus traditionnelle pour déterminer le droit applicable dans les relations de travail d'une entreprise québécoise de location de machinerie lourde et de transport routier intraprovincial $^{58}$. Quelques grues lui appartenant étaient utilisées pour effectuer du débardage auprès de navires. Même si c'était là une activité non significative de l'entreprise, cette dernière prétendait échapper de ce simple fait à la compétence provinciale. La Cour

53. Guy TREMBLAY, "L'application du droit provincial en matière maritime après l'affaire Succession Ordon ", (1999) 59 R. du B. 679. Voir aussi Henri Brun et Guy TREmBlay, Droit constitutionnel, 4e éd., Cowansville, Éditions Yvon Blais, 2002, pp. 487-491.

54. Banque canadienne de l'Ouest c. Alberta, [2007] 2 R.C.S. 3; ColombieBritannique (Procureur général) c. Lafarge Canada Inc. [2007] 2 R.C.S. 86.

55. R. c. Mersey Seafoods Ltd., (2008) NSCA 67; Jim Pattison Enterprises c. Workers' Compensation Board, 2011 BCCA 35 et Ryan Estate c. Universal Marine, (2009) NLTD 120.

56. Québec (Procureur général) c. Canadian Owners and Pilots Association, [2010] 2 R.C.S. 536.

57. Voir Christopher J. GiAschI, "Confused Seas : The Application of Provincial Statutes to Maritime Matters " publié sur le site web de Giaschi et Margolis (www.admiraltylaw.com).

58. Tessier Ltée c. Québec (CSST), préc., note 32. 
suprême, comme elle l'avait déjà fait par le passé59, a distingué entre une entreprise de transport maritime intraprovincial et une entreprise de transport maritime extraprovincial afin de déterminer qui, du Parlement du Canada ou de l'Assemblée législative d'une province, peut intervenir dans le champ des relations de travail. Même si la règle habituelle dans ce domaine est l'application du droit provincial, le tribunal avait jugé que le Parlement canadien peut réglementer le débardage puisqu'il s'agit là d'activités qui sont partie intégrante de la compétence fédérale en matière de navigation et marine marchande. Mais encore faut-il que cette activité soit une caractéristique essentielle de l'entreprise, ce qui n'était pas le $\operatorname{cas}^{60}$.

Mais à notre avis, l'arrêt Succession Ordon c. Grail rend l'application éventuelle du droit provincial quasi impossible en matière maritime, sauf exceptions ${ }^{61}$. D'abord, parce qu'il n'existe pas de "vide " à proprement parler en droit maritime canadien. La common law britannique supplée à l'absence de règles découlant de la législation fédérale ou de la jurisprudence canadienne. Et si une règle de droit britannique paraît obsolète, le tribunal peut toujours la modifier pour tenir compte de l'évolution du droit62. Puis parce que l'uniformité du droit maritime constitue le dogme sur lequel repose l'approche adoptée par la Cour suprême du Canada dans le domaine du droit maritime. Et c'est justement l'uniformité du droit maritime canadien qui plaide, selon la Cour, en faveur de l'exclusion de règles provinciales ${ }^{63}$.

59. Reference re Industrial Relations and Disputes Investigations Act, [1955] R.C.S. 529; Agence Maritime Inc. c. Le Conseil canadien des relations ouvrières, [1969] R.C.S. 851.

60. Dans R c. Navimez, [1998] R.J.Q. 1693 (C.A.), la Cour d'appel du Québec avait laissé entendre que certaines activités comme les rapports collectifs de travail au sein d'une entreprise qui organise des excursions d'observation de baleines dans les eaux du Québec pouvaient être régies par le droit provincial.

61. Préc., note 52.

62. Préc., note 51.

63. Voir André BRAËN, préc., note 32, pp. 501-509. 
Si l'uniformité peut être souhaitable, la réalité est tout autre. Ainsi, le texte d'une loi fédérale peut lui-même renvoyer à l'application du droit provincial64. Puis l'uniformité du droit au niveau international est loin d'être acquise, comme on l'a vu65, et elle ne signifie certainement pas là l'écart des traditions juridiques nationales même si elle invite ces dernières à concourir à la solution commune. De plus, les divers régimes juridiques applicables au transport maritime de biens ou de personnes distinguent entre la phase terrestre, antérieure et postérieure au transport, et celle proprement maritime et pour laquelle des règles différentes peuvent trouver application ${ }^{66}$. Finalement, les parties à un contrat maritime restent toujours libres, à moins qu'il ne s'agisse de règles d'ordre public, de choisir le droit applicable à leurs relations. Ainsi, rien n'empêche les parties à un contrat d'affrètement de prévoir le droit qui lui sera applicable et qui pourrait être dans notre cas le droit civil québécois ${ }^{67}$.

L'utilisation du droit civil n'aurait donc pas été complètement bannie du domaine maritime. De plus, l'exclusion du droit civil des affaires maritimes n'est pas absolue selon le professeur

64. Par exemple, la Loi sur les connaissements, L.R.C. 1985, c. B-5, tisse un lien contractuel entre le transporteur et le détenteur d'un connaissement même si ce dernier n'a pas été partie au contrat original de transport. L'article 3 renvoie spécifiquement à l'application du droit des provinces pour la détermination des droits de certains créanciers dont ceux du vendeur impayé sous le régime du Code civil. Voir aussi la Loi sur la responsabilité civile de l'État et le contentieux administratif, L.R.C. 1985, c. C-50 et la Loi sur les océans, L.C. 1996, c. 31.

65. Supra, remarques préliminaires.

66. Par exemple, les Règles de La Haye/Visby, applicables au Canada en vertu de la Loi sur la responsabilité en matière maritime, L.C. 2001, c. 6, art. 43, instaurent un régime obligatoire de responsabilité imputable au transporteur maritime. Mais ce régime peut être écarté en ce qui concerne les phases antérieure et postérieure du transport maritime à proprement parler (règles I(e) et VII).

67. Mais il faut avouer qu'à cet égard, les chartes-parties au Canada renvoient presque toujours à l'application du droit anglais. Par ailleurs, nous ne sommes pas certains que les dispositions du Code civil du Québec (art. 2001 à 2029) portant sur l'affrètement résisteraient à un examen constitutionnel de la part des tribunaux compte tenu de l'état actuel de la jurisprudence. 
(2013) 43 R.D.U.S. rôle du droit civil québécois
et l'utilisation du droit comparé
en droit maritime canadien

Glenn puisque certains principes mis de l'avant par l'Amirauté britannique sont d'inspiration civiliste, comme c'est le cas avec les privilèges maritimes 68 . Sa pertinence se situe donc au niveau de la preuve de l'incorporation d'un principe civiliste par la Cour d'Amirauté. Si historiquement c'était le cas, il reste qu'aujourd'hui, le recours au droit civil par les tribunaux anglais nous apparaît quand même exceptionnel. Dans l'arrêt Q.N.S. Paper Co. c. Chartwell Shipping Ltd., la majorité a reconnu l'utilité de la méthode comparative et la prise en considération de principes civilistes pour régler un litige maritime, même si elle n'y a pas fait appel pour régler les questions en litige (ayant trait à la responsabilité du mandant), cette analyse s'étant faite exclusivement sous l'angle de la common law69. Dans la même décision, la juge L'Heureux-Dubé a plaidé, quant à elle, pour une application plus systématique en droit maritime canadien de la méthode comparative qui fait appel à l'analyse de principes civilistes. La Cour d'Amirauté anglaise utilisait de tels principes et ces derniers ont été incorporés dans le droit canadien en 1934. En permettant de considérer des principes civilistes, la méthode comparative vise donc à établir une harmonie qui n'atteint toutefois pas l'homogénéité. La méthode permet de clarifier des problèmes juridiques communs aux deux traditions juridiques en exploitant les différences doctrinales et méthodologiques ${ }^{70}$. La clarté du raisonnement juridique est favorisée quand un même problème est envisagé de plusieurs points de vue différents. La méthode comparative vise pardessus tout à multiplier le nombre de sources auxquelles on peut puiser aux fins d'une analyse juridique. Bref, elle permet une analyse plus poussée. La juge McLachlin a reconnu, quant à elle, l'utilité de la méthode comparative et la prise en considération des principes civilistes et s'est même demandé si compte tenu d'une

68. H.P. GLenN, "Maritime Law-Federal Court Jurisdiction - Canadian Maritime Law - Relationship to Civil and Common Law : Ito-International Terminal Operators Ltd. v. Miida Electronics Inc. ", (1987) 66 R. du B. can. 360 et E.F. RYAN, "Admiralty Jurisdiction and the Maritime Liens: An Historical Perspective ", (1968) 7 Western Ont. Law Review, 173.

69. Préc., note 18.

70. Id., pp. 717-721. 
compétence illimitée, des principes autres que de common law pourraient s'appliquer en droit maritime canadien ${ }^{71}$. En conclusion, l'utilité du droit civil en droit maritime canadien se résume aujourd'hui à constituer un support comparatif utile pour déterminer, s'il y a lieu, les règles de common law applicables à un litige maritime.

\section{III- Le rôle du droit civil}

Toutefois, c'est surtout au niveau de la troisième étape dégagée dans l'arrêt Succession Ordon c. Grail, traitant de la réforme du droit par les tribunaux, que l'on appréhende mieux le rôle désormais dévolu au droit civil dans la résolution d'une affaire maritime instruite par les tribunaux canadiens et québécois. C'est lorsque le tribunal au Canada doit se demander s'il ne convient pas de modifier une règle de droit maritime non législative. En l'absence d'une règle législative fédérale ou de droit maritime proprement dit, c'est à la règle non législative qu'il faut s'en remettre pour solutionner un litige maritime. Par là, on entend la règle de common law appliquée par les tribunaux anglais dans un litige similaire. Si cette règle apparaît trop ancienne, il faut alors vérifier s'il n'y a pas lieu pour le tribunal canadien de la réformer pour assurer l'évolution du droit. Une analyse chronologique de la jurisprudence de la Cour suprême du Canada à cet égard permet d'avancer le constat suivant :

- Dans l'arrêt Norsk ${ }^{72}$, la Cour suprême du Canada a vérifié quelles étaient les règles de common law applicables à l'indemnisation des pertes économiques et qui requerraient une modification par la cour. Après une analyse du droit applicable dans les juridictions de common law, la majorité du tribunal avait aussi noté qu'en droit civil, aucune distinction n'était faite entre préjudice physique et préjudice économique et que l'application

71. Id., p. 692.

72. Cie des chemins de fer nationaux du Canada c. Norsk Pacific Steamship Co., [1992] 1 R.C.S. 1021. 
des règles générales de la responsabilité civile n'avait pas conduit à un système de responsabilité illimitée dans ce domaine ${ }^{73}$.

- Dans l'arrêt Bow Valley Husky (Bermuda)74, la Cour suprême voulait réformer les règles de common law applicables à un cas de négligence contributive en matière maritime. L'examen des solutions offertes par les droits anglais, australien, américain et aussi celles applicables dans les provinces de common law et celles offertes tant par le droit civil québécois que français fut jugé très utile par la juge McLachlin.

- Dans l'arrêt Succession Ordon 75 , la modification de la règle de common law empêchant l'indemnisation des personnes à charge d'une victime a été revue en considérant l'état du droit dans les provinces de common law mais non du Québec. En ce qui concerne la règle interdisant l'indemnisation pour pertes de conseils, de soins et de compagnie, la Cour suprême a pris en considération, en plus de la common law canadienne-anglaise, le droit du Royaume-Uni et aussi le Code civil du Québec.

- Dans l'arrêt Fraser River Pile \& Dredge ${ }^{76}$ et pour vérifier le droit d'un tiers à utiliser une clause contractuelle compte tenu de la règle du lien contractuel, la Cour suprême a aussi considéré le droit civil en plus de faire un examen exhaustif du droit applicable par les juridictions de common law ${ }^{77}$.

73. Pour une analyse de cette problématique, voir André BRAËN, "L'indemnisation de la perte économique en droit maritime canadien ", (1997) XV Annuaire de droit océanique et maritime, 31 et "L'uniformisation du droit maritime canadien - les cas de l'indemnisation d'une perte économique et de la négligence contributive l'arrêt Husky Oil Operations Ltd. c. Saint-John Shipbuilding Ltd. ", (1998) 77 R. du B. can. pp. 509.

74. $\quad$ Préc., note 18.

75. $\quad$ Préc., note 18.

76. Fraser River Pile \& Dredge Ltd. c. Can-Dive Services Ltd., [1999] 3 R.C.S. 108.

77. Il est à noter que dans cette affaire en provenance de la ColombieBritannique, la Cour suprême ne procède même pas à la qualification du litige comme étant une question maritime devant être régie par le droit maritime canadien. Un oubli? 
L'utilisation de la méthode comparative par la Cour suprême n'est pas systématique. D'abord, dans l'arrêt ITO International Terminal Operators ${ }^{78}$, la majorité du tribunal a analysé la validité en droit maritime canadien de la "clause Himalaya "79 en fonction du droit des seules juridictions de common law. Dans l'arrêt Porto Seguro Companhia de Seguros Gerais ${ }^{80}$, la règle interdisant le témoignage d'experts quand des assesseurs sont nommés par un tribunal fait l'objet d'une brève considération par la Cour suprême. Celle-ci analyse les règles applicables à ce sujet dans les provinces de common law mais non au Québec. Dans l'arrêt Armada Lines Ltd. ${ }^{81}$, le même tribunal s'est demandé s'il y avait lieu de changer la règle de common law accordant des dommages-intérêts à la suite d'une saisie illégale uniquement dans les cas de mauvaise foi. L'analyse de la Cour se fait sous l'angle exclusif de la common law. Enfin, il faut garder en mémoire que lorsque la Cour suprême utilise l'approche comparative, son analyse de la solution provenant du droit civil n'est pas, bien souvent, développée. En effet, le tribunal se contente simplement de renvoyer à certains articles du Code civil, laissant ainsi le comparatiste sur sa faim.

\section{Conclusion}

En interdisant le recours à titre supplétif au droit commun des provinces et donc au droit civil québécois, la démarche judiciaire adoptée par la Cour suprême du Canada a rompu l'unité du droit et du même coup, a rendu le droit maritime canadien incertain. Elle le dissocie et l'isole artificiellement en le traitant comme un corps de règles complet et autonome. Pourtant, il est évident que le droit maritime canadien ne peut se suffire totalement à lui-

78. Préc., note 18.

79. Cette clause permet au manutentionnaire de marchandises de pouvoir invoquer à son bénéfice des clauses de non-responsabilité contenues dans le contrat de transport maritime dont il n'est pas partie. C'est le principe du lien contractuel en common law qui est alors en cause.

80. Porto Seguro Companhia de Seguros Gerais c. Belcan S.A., [1997] 3 R.C.S. 1278.

81. Armada Lines Ltd. c. Chaleur Fertilizers, [1997] 2 R.C.S. 617. 
même. Par exemple, si le droit maritime canadien détermine les droits et obligations des parties à un contrat de transport sous connaissement ${ }^{82}$, il reste silencieux quant aux conditions de fond relatives à la validité dudit contrat (capacité, consentement...) et normalement, c'est le droit commun qui serait appelé à intervenir pour résoudre ces questions, ne serait-ce qu'à titre supplétif. Mais l'approche judiciaire fait fi de cette réalité et elle invite le Parlement canadien à intervenir fréquemment pour suppléer à cette lacune. Ce dernier doit lentement élaborer un droit privé fédéral en matière maritime. C'est ainsi que, par exemple, le Parlement dut intervenir en 2009 pour amender sa Loi sur la responsabilité en matière maritime ${ }^{83}$ afin de préciser qu'à moins de dispositions contraires, toute action qui se rapporte au droit maritime canadien doit se prescrire par trois ans à compter du fait générateur du litige ${ }^{84}$.

Cette approche judiciaire a donc réduit le rôle du droit civil québécois en matière maritime à celui d'un simple support comparatif, y inclus sur son territoire naturel, et ce, sur le même pied que tout autre droit étranger. Et lorsque la méthode comparative est employée, la référence reste souvent sommaire et peu élaborée. "Le droit civil n'intervient qu'à travers le filtre de la common law ou du droit comparé ", écrivait le juge Lebel ${ }^{85}$ parlant ainsi de la dissociation en droit maritime entre la législation fédérale et le droit provincial, une dissociation créée et voulue, semble-t-il, par le Parlement canadien. La scission entre le droit civil québécois et le droit maritime canadien est telle qu'il s'agit, selon le juge, d'une exception à l'interaction entre le droit civil et la common law que normalement le Parlement et la Cour suprême veulent harmonieuse ${ }^{86}$.

82. Loi sur la responsabilité en matière maritime, préc., note 38, art. 43. Cette législation adoptée en 2001 est elle-même en partie le résultat de cette approche judiciaire.

83. Préc., note 38, art. 140.

84. L.C. 2009, c. 21 , art. 12.

85. Louis LEBEL, "L'interaction du droit civil et de la common law à la Cour suprême du Canada ", (2006) 47 C. de D. 179, p. 236.

86.

$$
\text { Id. }
$$


Il est peut-être vrai qu'à l'origine de cette attribution d'une compétence maritime à la Cour fédérale, le Parlement ait voulu créer au Canada un tribunal d'amirauté capable de régler tout litige maritime en lui attribuant, pour ce faire, une compétence judiciaire semblable à celle qu'aurait exercée l'amirauté anglaise n'eût été des limites que les cours de common law lui avaient imposées au fil des siècles. Mais le Parlement canadien a-t-il ce pouvoir? L'Angleterre est un État unitaire et connaît une tradition juridique. Le Canada est un État fédéral où coexistent deux traditions juridiques. Malheureusement, cette question n'a jamais été abordée par la Cour suprême du Canada. Il est ironique de constater dans un congrès de droit comparé que l'approche comparative adoptée par la Cour suprême qui permet le recours au droit civil québécois dans le domaine maritime sert simplement à en atténuer le retrait.

À notre avis, cette approche judiciaire qui, à titre de prix de consolation, attribue au droit civil québécois un simple rôle comparatif en droit maritime canadien est inacceptable et ne sert, selon un auteur87, qu'à renforcer "les solitudes du bijuridisme canadien ". Le Parlement canadien dans sa volonté d'harmoniser sa législation avec le droit civil québécois a, malheureusement et à deux reprises, manqué le bateau en confirmant dans le domaine maritime ce rôle dévolu au droit civil ${ }^{88}$. Mais le Parlement veut-il vraiment modifier une approche judiciaire qui, dans le domaine du partage des compétences, lui reconnaît à toute fin pratique et malgré le cadre fédéral des pouvoirs illimités?

87. Jean-François Gaudreault-Desbiens, Les solitudes du bijuridisme au Canada, Montréal, Éditions Thémis, 2007.

88. Loi d'harmonisation no 1 du droit fédéral avec le droit civil, L.C. 2001, c. 4 et Loi d'harmonisation no 2 du droit fédéral avec le droit civil, L.C. 2004, c. 25. Cette législation a pour objet de faire du droit provincial en matière de propriété et de droits civils le droit supplétif pour ce qui est de la législation fédérale dans les provinces sauf règle de droit s'y opposant. Évidemment, l'approche de la Cour suprême du Canada dans le domaine maritime constitue une règle de droit s'opposant à l'application du droit civil. 